\title{
Information technologies of activation of informative activity of students
}

\author{
L.R. Sharifullina ${ }^{1}$, V.A. Tytar ${ }^{1, *}$ \\ ${ }^{1}$ Moscow Technological Institute, 199334, Moscow, Russia
}

\begin{abstract}
The paper describes the aspects of the augmented reality application development on the base of ARToolkit for Android. The steps consistently used for the augmented reality application development have been considered. The simplest Java-based examples of augmented reality have been discussed.
\end{abstract}

\section{Introduction}

It is impossible to deny the widespread penetration of information technologies and their profound influence on many spheres of human activity.

The last 10-15 years in Russia as in the rest of the world, successful business is not possible without the use of email or Internet advertising [1]. An even more striking example of the infusion of the Internet in human activity is dramatically increased over the past 5 years, the interest in online games. Has been spared and educational (training) activities. In education an important role is played by information and communication technologies. This is evidenced by their widespread use and implementation in the curricula and structure of the leading universities of Europe and Russia. Almost all of these traditional universities make certain steps towards the systematic use of information technologies [2].

Under the traditional institutions of higher education is to understand higher education institutions, adhering to historically established norms of teaching and the organization of the educational process within its structure: lectures and seminars, carrying out practical and laboratory work, monitoring attendance, etc.

\section{Analysis and discussion}

Traditional higher educational establishment is characterized by the applicability in the organization and analysis of the educational process traditional pedagogical categories, such as objectives, form, content, and teaching methods.

Available pedagogical tools, up to a certain time, is quite enough for the realization of set educational objectives of the traditional higher educational establishment (Institute, University, Academy). But referring to the didactic prerequisites for the implementation of eLearning systems, it is worth noting an interesting fact: the vast majority of teachers are always very skeptical of pedagogy, as science sometimes does it as such without taking.

It was caused primarily by the fact that one of the basic concepts of pedagogy "technique" is characterized by a high degree of subjectivity, due to the inaccuracies of many of the definitions and wording. And because the technique in itself describes the key points of the training activities and the relationship of the educational objectives, forms, methods and means of education.

Modern pedagogy is currently experiencing is also a certain "human resources" crisis. The vast majority of current teachers have been trained and trained in the Soviet period, taking into account those realities, living standards and achievements of science and technology.

The student of today is the man who is perfectly fluent in modern communication tools, advanced user of personal computer and other electronic equipment and, as a rule, easily trainable and communicative. Its ability to absorb and assimilate information very high, but sharply raises the question about the sources of knowledge and skills.

All students spend more time on the Internet: searching for information, doing work or communicating with peers and. At the same time, the rapidly changing modern world sets the need for more rapid creation of new knowledge and their further transfer. In an attempt to solve this problem, it was proposed the introduction in the educational process of e-learning systems.

Under the e-learning system we understand the educational process with the use of modern information technologies and electronic UNESCO experts have given this phenomenon the name «E-learning», defined as "learning through the Internet and media." This term, in fact, is an extended definition of "distance learning."

For e-learning is characterized by a number of features which distinguishes it from the traditional educational technologies:

- independent work of students with electronic learning materials;

* Corresponding author: $\underline{\mathrm{v} \text { titar@mti.edu.ru }}$ 
- the possibility of on line consultation and webinars professionals located remotely from the place of employment;

- the ability to create information online - communities (forums, social networks), with the aim of educational communication and management of virtual training activities;

- availability of server-based storage of learning content: books, guidelines, both for students and for teachers, presentations, handouts, etc .;

- access to information regardless of time and place, if you have access to the access to the Internet;

- ability to replenish resources and making them relevant adjustments;

- the possibility of training disabled persons.

The use of this training system contributes to the development of information culture of students and teachers, enabling them to master modern pedagogical technologies and improve the efficiency of their work.

The most popular and effective electronic products used for training in Russian universities, became a Web program «Moodle», which is a modular dynamic learning environment. It is known as education management system.

The system is focused on the organization of interaction between teachers and students, as well as the transfer of knowledge in the form of electronic files. It can also be used in the construction of traditional classes or distance learning.

Ease of use and the ability to self-replenish an information bank, depending on the subject of specialization, provided the application «Moodle» widespread popularity in more than one hundred and fifty countries.

Because the application has an advanced modular architecture, the capabilities "Moodle" can simply be extended and added by third-party developers. A significant expansion of the functionality of "Moodle" is possible in the use of the integration of a subsystem for the organization of webinars (web - conferences).
Teachers can prepare and provide students with information in electronic form, by placing it in a special portals storage. There also may be placed for the job knowledge test: test papers, case studies, assignments, etc. Students, in turn, can engage in the educational process at a convenient time for them, as well as to communicate with the teachers in the on-line and send them to perform tasks in the form of a text's documents, images

\section{Conclusions}

Summarizing all the above, we turn to sociology, namely the theory of knowledge developed by Peter Berger and Thomas Luckmann, called "social constructionism". According to this theory, individuals and groups of people are involved in the creation of their perceived social reality. One of the tasks of the social constructionism becomes a study of the processes by which man creates, realizes and implements social phenomena in tradition.

$\mathrm{n}$ other words, we create the conditions of their social behavior, every day, performing the same actions, guided by certain rules. But the so-called "social constructs" is an object of knowledge, not specified initially from nature. And their continued existence, they must be constantly maintained, upgraded and evolve with the changes and dynamic educational environment needs. Only the systematic use of e-learning systems, as the most interesting non-traditional technologies, allow a different look at the issue of teaching many subjects, and to make the process of learning to a new level

\section{References}

1. L. Sharifullina, I. Cherkashin. Modern problems of science and education. 6, 38 (2013)

1. I. Cherkashin, L. Sharifullina. Scientific and educational civil protection issues, 93 (2013) 